\title{
ESBL-producing E. coli and K. pneumoniae in Al-Ahsa, Saudi Arabia: antibiotic susceptibility and prevalence of $b / a_{\mathrm{SHV}}$ and $b / a_{\mathrm{TEM}}$
}

\author{
Abdulrahman A. Alsultan ${ }^{1}$, Elsayed Aboulmagd ${ }^{1,2}$, Tarek T. Amin $^{3}$ \\ ${ }^{1}$ Department of Biomedical Sciences, Division of Medical Microbiology, College of Medicine, King Faisal University, \\ Al Ahsa, Kingdom of Saudi Arabia \\ ${ }^{2}$ Department of Microbiology, Faculty of Pharmacy, Alexandria University, Alexandria, Egypt \\ ${ }^{3}$ Family and Community Medicine Department, College of Medicine, King Faisal University, Al Ahsa, Kingdom of \\ Saudi Arabia
}

Key words: ESBL; E. coli; K. pneumoniae; antimicrobial susceptibility; $b_{\mathrm{SHV}}$, bla $a_{\mathrm{TEM}}$

J Infect Dev Ctries 2013; 7(12):1016-1019. doi:10.3855/jidc.3764

(Received 06 May 2013 - Accepted 26 September 2013)

Copyright (C) 2013 Alsultan et al. This is an open-access article distributed under the Creative Commons Attribution License, which permits unrestricted use, distribution, and reproduction in any medium, provided the original work is properly cited.

\section{Introduction}

Antibiotic resistance is a serious problem in clinical medicine [1]. Production of inactivating enzymes is one of the most common mechanisms by which different microorganisms can acquire resistance against several antimicrobial agents, especially $\beta$ lactams. Extended spectrum $\beta$-lactamases (ESBLs) mediate resistance against different broad spectrum cephalosporins and monobactams [2].

There are different types of ESBLs, namely TEM, SHV, and CTX, but most of these enzymes are mutants of TEM1, TEM2, and SHV1 that are caused by point mutation of the original encoding genes [3]. These genes are carried on plasmids that may harbor other genes encoding resistance against different antimicrobial agents. These plasmids are easily transferred among different bacterial species, leading to widespread of multi-drug resistant bacteria [4]. E. coli and Klebsiella spp. are the most common producers of ESBLs and are responsible for many hospital- and community-acquired infections. Due to the extensive use of cephalosporins for treatment of different bacterial infections, the prevalence of ESBLproducing bacteria became significantly high worldwide [5].

In Saudi Arabia, limited data are available on the susceptibility patterns of ESBL-producing bacteria. The aim of the current study was to evaluate the susceptibility of ESBL-producing $K$. pneumoniae and $E$. coli clinical isolates to different antimicrobial agents and to estimate the prevalence of $b l a_{\mathrm{SHV}}$ and bla $_{\mathrm{TEM}}$ in the tested isolates.

\section{Methodology}

A total of 97 non-duplicate ESBL-producing clinical isolates were collected from the Al-Ahsa region of Saudi Arabia. The isolates were identified by the Vitek 2 compact automated system (BioMerieux, Marcy L'Etoile, France) as $K$. pneumoniae (37 isolates) and E. coli (60 isolates) using GN cards.

The minimum inhibitory concentrations (MICs), resistance patterns, and preliminary phenotypic detection of ESBL production were determined using the Vitek 2 compact automated system using ASTN116 cards. Confirmation of ESBL production was done using the following Etest ESBL-strips (AB Biodisk, Solna, Sweden): Cefotaxime / cefotaxime plus clavulanic acid (CT/CTL), ceftazidime / ceftazidime plus clavulanic acid (TZ/TZL) and cefepime / cefepime plus clavulanic acid (PM/PML).

Polymerase chain reaction (PCR) amplification was used to detect the presence of genes encoding TEM and SHV enzymes as previously described [6]. For amplification of bla $_{\mathrm{TEM}}$, the following primers were used: 5'-AGATCAGTTGGGTGCACGAG-3' and 5'-CAGTGCTGCAATGATACCGC-3', while $b l a_{\text {SHV }}$ was detected using the following primers: $5{ }^{\prime}-$ CGCCTGTGTATTATCTCCC-3' and 5'GGCGATTTGCTGATTTCGC-3`. 
Table 1. Antimicrobial susceptibility patterns of ESBL-producing E. coli and K. pneumoniae isolates

\begin{tabular}{|c|c|c|c|c|c|c|}
\hline \multirow{3}{*}{ Antimicrobial agents } & \multicolumn{3}{|c|}{ Escherichia coli $(\mathrm{n}=60)$} & \multicolumn{3}{|c|}{ Klebsiella pneumoniae $(\mathrm{n}=37)$} \\
\hline & Susceptible & Intermediate & Resistant & Susceptible & Intermediate & Resistant \\
\hline & \multicolumn{3}{|c|}{$n(\%)$} & \multicolumn{3}{|c|}{$n(\%)$} \\
\hline Ampicillin & $0(0)$ & $0(0)$ & $60(100)$ & $0(0)$ & $0(0)$ & $37(100)$ \\
\hline Ampicillin/Sulbactam & $6(10)$ & $17(28.3)$ & $37(61.7)$ & $2(5.4)$ & $9(24.3)$ & $26(70.3)$ \\
\hline Piperacillin & $0(0)$ & $0(0)$ & $60(100)$ & $0(0)$ & $0(0)$ & $37(100)$ \\
\hline Piperacillin/Tazobactam & $48(80)$ & $6(10)$ & $6(10)$ & $17(45.9)$ & $4(10.8)$ & $16(43.2)$ \\
\hline Cefazolin & $0(0)$ & $0(0)$ & $60(100)$ & $0(0)$ & $0(0)$ & $37(100)$ \\
\hline Cefuroxime & $0(0)$ & $0(0)$ & $60(100)$ & $0(0)$ & $0(0)$ & $37(100)$ \\
\hline Cefuroxime Axetil & $0(0)$ & $0(0)$ & $60(100)$ & $0(0)$ & $0(0)$ & $37(100)$ \\
\hline Cefoxitin & $42(70)$ & $6(10)$ & $12(20)$ & $24(64.9)$ & $4(10.8)$ & $9(24.3)$ \\
\hline Cefpodoxime & $0(0)$ & $0(0)$ & $60(100)$ & $0(0)$ & $0(0)$ & $37(100)$ \\
\hline Cefotaxime & $11(18.3)$ & $8(13.3)$ & $41(68.3)$ & 7 (18.9) & $5(13.5)$ & 25 (67.6) \\
\hline Ceftazidime & $21(35)$ & $34(56.7)$ & $5(8.3)$ & $5(13.5)$ & $15(40.5)$ & 17 (45.9) \\
\hline Cefepime & $46(76.7)$ & $2(3.3)$ & $12(20)$ & $24(64.9)$ & $5(13.5)$ & $8(21.6)$ \\
\hline Imipenem & $60(100)$ & $0(0)$ & $0(0)$ & $37(100)$ & $0(0)$ & $0(0)$ \\
\hline Meropenem & $60(100)$ & $0(0)$ & $0(0)$ & $37(100)$ & $0(0)$ & $0(0)$ \\
\hline Gentamicin & $22(36.7)$ & $0(0)$ & $38(63.3)$ & $16(43.2)$ & $0(0)$ & $21(56.8)$ \\
\hline Tobramycin & $15(25)$ & $13(21.6)$ & $32(53.4)$ & 7 (18.9) & $14(37.8)$ & $16(43.2)$ \\
\hline Ciprofloxacin & $13(21.7)$ & $1(1.6)$ & $46(76.7)$ & $6(16.2)$ & $5(13.5)$ & $26(70.3)$ \\
\hline Levofloxacin & $14(23.3)$ & $0(0)$ & $46(76.7)$ & $8(21.6)$ & $8(21.6)$ & $21(56.8)$ \\
\hline Tigecycline & $60(100)$ & $0(0)$ & $0(0)$ & $27(73.0)$ & $4(10.8)$ & $6(16.2)$ \\
\hline Sulphamethoxazole/Trimethoprim & $13(21.7)$ & $0(0)$ & $47(78.3)$ & 7 (18.9) & $0(0)$ & $30(81.1)$ \\
\hline
\end{tabular}

Table 2. Prevalence of bla $a_{\mathrm{SHV}}$ and bla ${ }_{\mathrm{TEM}}$ in ESBL-producing E. coli and K. pneumoniae isolates

\begin{tabular}{lccc}
\hline \multirow{2}{*}{ ESBL encoding gene } & $\begin{array}{c}\text { Escherichia coli } \\
\text { No }(\%), 95 \% \text { CI }\end{array}$ & $\begin{array}{c}\text { Klebsiella pneumoniae } \\
\text { No }(\%), 95 \% \text { CI }\end{array}$ & P value * \\
\hline bla $_{\mathrm{SHV}}$ & $20(33.3), 22.7-45.9$ & $29(78.4), 62.8-88.6$ & 0.001 \\
bla $_{\mathrm{TEM}}$ & $44(73.3), 58.7-80.8$ & $16(43.2), 28.7-59.1$ & 0.005 \\
bla $_{\mathrm{SHV}}$ and $b l a_{\mathrm{TEM}}$ & $11(18.3), 10.6-29.9$ & $8(21.6), 11.4-37.2$ & 0.894 \\
\hline$*$ Z test for proportions (two ways) & &
\end{tabular}

Data analysis was done using SPSS version 16.0 (SPSS Inc, Chicago, USA). For categorical data, frequency, proportions, and percentage were used for reporting, and the $\mathrm{Z}$ test for proportions was used for comparison. Univariate analysis with estimation of odds ratio (95\% confidence intervals) was also used for comparison. For continuous data mean, standard deviation and median were used for expression. A pvalue of $<0.05$ was used as the level of statistical significance.

\section{Results and Discussion}

The majority of the isolates were collected from urine specimens (35 E. coli and $14 \mathrm{~K}$. pneumoniae) while only small numbers of isolates (two E. coli and one $K$. pneumoniae) were obtained from catheter tips. The rest of the isolates were collected from infected wounds (16 E. coli and $15 \mathrm{~K}$. pneumoniae), from respiratory tracts, (four $E$. coli and six $K$. pneumoniae), and from blood (three $E$. coli and one $K$. pneumoniae). No significant difference was found in the prevalence of both pathogens recovered from different clinical specimens. Comparable patterns of microbial isolates were previously reported from different areas of Saudi Arabia [7,8].

The Vitek 2 compact system was used for detection of the ESBL-producing isolates and for confirmation of ESBLs production, three Etest ESBLstrips were used: CT/CTL, TZ/TZL, and PM/PML. The most sensitive strip was PM/PML, followed by CT/CTL; while TZ/TZL was the least sensitive one (data not shown). Similar results were previously published [9] showing that the PM/PML strip was the best configuration and the most suitable substitute for detection of ESBLs.

The antimicrobial susceptibility patterns of the tested isolates were depicted in Table 1. All isolates were resistant to ampicillin, piperacillin, cefazolin, cefuroxime, and cefpodoxime. Incorporation of tazobactam strongly affected the susceptibility of many tested isolates to piperacillin (67\%) while sulbactam showed slight ability to reverse the 
resistance of most isolates to ampicillin (8\%). These results are consistent with recently published data [8].

The current study showed that carbapenems (imipenem and meropenem) were active against all tested isolates. Similar susceptibility to carbapenems $(100 \%)$ was reported earlier [10,11]. In contrast, reduced susceptibility to imipenem and meropenem was found previously [12,13]. Nevertheless, carbapenems are still the drug of choice for the treatment of life-threatening enterobacterial infections caused by ESBL-producing pathogens [3]. Recently, many reports showing high levels of resistance to carbapenems were published [12,13]. Therefore, seeking out of alternatives that show in vitro activity against ESBL-producing pathogens (e.g., fluoroquinolones, tigecycline, or antibiotic combinations) is important.

Tigecycline exhibited activity against all tested $E$. coli strains (MICs values were $\leq 4$ ). Variable susceptibility was noted with $K$. pneumoniae isolates - susceptible $(73 \%)$, intermediate $(10.8 \%)$, and resistant $(16.2 \%)$. Previously published reports showed that tigecycline exhibited excellent activity against ESBL-producing E. coli and $K$. pneumoniae $[7,8]$. Therefore, piperacillin-tazobactam and tigecycline hold promise to be alternatives that could limit the evolution and spread of carbapenem resistance.

Resistance to non $\beta$-lactam antimicrobial agents and third-generation cephalosporins is increasing globally. In this study, a high resistance rate was observed among tested isolates against sulphamethoxazole-trimethoprim (79\%), ciprofloxacin (74\%), levofloxacin $(69 \%)$, gentamicin $(61 \%)$, and tobramycin $(49.5 \%)$. Co-resistance to fluoroquinolones, aminoglycosides, and sulphamethoxazole-trimethoprim was detected in sixteen E. coli and nine $K$. pneumoniae isolates (data not shown). In addition, four Klebsiella pneumoniae strains showed resistance to cefepime in addition to the three previously mention antimicrobial groups. Variable levels of resistance were reported in different local and regional studies $[7,8,11,13]$. This variation in the resistance/susceptibility patterns may be due to the types of antimicrobial agents commonly used in certain areas and the rate at which antibiotics are prescribed for treatment of various infectious diseases. Therefore, clinicians should be familiar with the antimicrobial stewardship programs to promote the optimum usage of antimicrobial agents (type, dose, duration, and route of administration) [14].
The prevalence of $b l a_{\mathrm{SHV}}$ and $b l a_{\mathrm{TEM}}$ in the tested isolates is summarized in Table 2. Bla $a_{\mathrm{SHV}}$ was significantly dominant in $K$. pneumoniae isolates (78.4\%). This result agreed with recently published data from Saudi Arabia $[8,15]$. On the other hand, $b l a_{\text {TEM }}$ was found to be highly prevalent in E. coli isolates $(73.3 \%)$ in comparison with $K$. pneumoniae $(43.2 \%)$. The prevalence of bla $_{\mathrm{TEM}}$ in Enterobacteriaceae differs across regions in Saudi Arabia $[8,15]$. Both $b l a_{\mathrm{SHV}}$ and $b l a_{\mathrm{TEM}}$ were coexistent in eleven (18\%) E. coli and eight (22\%) $K$. pneumoniae isolates, which was statistically insignificant. The detected $b l a_{\mathrm{SHV}}$ and $b l a_{\mathrm{TEM}}$ should be sequenced to explore the most prevalent type of $\beta$ lactamase genes in tested clinical isolates.

\section{Conclusion}

Because hospital settings are hotspots for the transmission of antibiotic resistance genes, a strict hospital infection control policy should be implemented; regular surveillance of microbial resistance is crucially needed. To ensure the judicious use of antibiotics, the local and regional data of resistance/susceptibility patterns should be available to clinicians, and effective local antibiotic policies should be applied. To limit the spread of multi-drug resistant pathogens, clinicians should test for ESBL-producing microorganisms along with the routine antimicrobial sensitivity testing they perform.

\section{Acknowledgements}

The authors are grateful for financial support of the deanship of scientific research of King Faisal University.

\section{References}

1. Choudhury R, Panda S, Singh DV (2012) Emergence and dissemination of antibiotic resistance: A global problem. Indian J Med Microbiol 30: 384-390.

2. Savard P, Perl TM (2012) A call for action: managing the emergence of multi-drug Enterobacteriaceae in acute care settings. Curr Opin Infect Dis 25: 371-377.

3. Paterson DL, Bonomo RA (2005) Extended-spectrum betalactamases: a clinical update. Clin Microbiol Rev 18: 657686.

4. Davies J, Davies D (2010) Origin and evolution of antibiotic resistance. Microbiol Mol Biol Rev 74: 417-433.

5. Savard P, Perl TM (2012) A call for action: managing the emergence of multi-drug Enterobacteriaceae in acute care settings. Curr Opin Infect Dis 25: 371-377.

6. Garza-González E, Mendoza Ibarra SI, Llaca-Díaz JM, Gonzalez GM (2011) Molecular characterization and antimicrobial susceptibility of extended-spectrum $\beta$ lactamase-producing Enterobacteriaceae isolates at a tertiary care centre in Monterrey, Mexico. J Med Microbiol 60: 8490 . 
7. Jamal WY, Al Hashem G, Khodakhast F, Rotimi VO (2009) Comparative in vitro activity of tigecycline and nine other antibiotics against gram-negative bacterial isolates, including ESBL-producing strains. J Chemother 21: 261-266.

8. Hassan MI, Alkharsah KR, Alzahrani AJ, Obeid OE, Khamis AH, Diab A (2013) Detection of extended spectrum betalactamases-producing isolates and effect of AmpC overlapping. J Infect Dev Ctries 7: 618-629.

9. Mohanty S, Gaind R, Ranjan R, Deb M (2009) Use of the cefepime-clavulanate ESBL Etest for detection of extendedspectrum beta-lactamases in AmpC co-producing bacteria. $\mathrm{J}$ Infect Dev Ctries 4: 24-29.

10. Khanfar HS, Bindayna KM, Senok AC, Botta GA (2009) Extended spectrum beta-lactamases (ESBL) in Escherichia coli and Klebsiella pneumoniae: trends in the hospital and community settings. J Infect Dev Ctries 3: 295-299.

11. Mehrgan H, Rahbar M, Arab-Halvaii Z (2010) High prevalence of extended-spectrum beta-lactamase-producing Klebsiella pneumoniae in a tertiary care hospital in Tehran, Iran. J Infect Dev Ctries 4: 132-138.

12. Centers for Disease Control and Prevention, CDC (2013) Vital signs: carbapenem-resistant enterobacteriaceae. MMWR Morb Mortal Wkly Rep 62: 165-170.
13. Zowawi HM, Balkhy HH, Walsh TR, Paterson DL (2013) $\beta$ Lactamase production in key gram-negative pathogen isolates from the Arabian Peninsula. Clin Microbiol Rev 26: 361-380.

14. Ashiru-Oredope D, Sharland M, Charani E, McNulty C, Cooke J, ARHAI Antimicrobial Stewardship Group (2012) Improving the quality of antibiotic prescribing in the NHS by developing a new Antimicrobial Stewardship Programme: Start Smart--Then Focus. J Antimicrob Chemother 67: 51-63.

15. Tawfik AF, Alswailem AM, Shibl AM, Al-Agamy MH (2011) Prevalence and genetic characteristics of TEM, SHV, and CTX-M in clinical Klebsiella pneumoniae isolates from Saudi Arabia. Microb Drug Resist 17: 383-388.

\section{Corresponding author}

Elsayed Aboulmagd

Division of Medical Microbiology, Department of Biomedical

Sciences

College of Medicine, King Faisal University, Saudi Arabia

Phone: +966-541870557

Fax: +966-3-5800820 (3030)

E-mail: elsayed20@hotmail.com

Conflict of interests: No conflict of interests is declared. 